\title{
REFUGIADOS, DESPLAZADOS E INMIGRANTES ECONÓMICOS. EL CASO DE LA DIGNIDAD ${ }^{1}$
}

\section{Refugiados, Pessoas Deslocadas e Migrantes Econômicos. O Caso da Dignidade}

\author{
Refugees, Displaced Persons and Economic \\ Migrants. The Case of the Dignity
}

Daniel Loewe

\section{Resumen}

El artículo analiza cómo se relaciona la premisa normativa de la dignidad humana con la evaluación del fenómeno migratorio. Para aquello, da cuenta de una concepción de dignidad humana que no recurre a presupuestos naturalistas ni supraempíricos, y presenta tres alternativas diferentes para otorgarle contenido normativo en base a un umbral suficientarista (la teoría de las relaciones internacionales de Rawls, la sociedad decente de Margalit, y el enfoque en las capacidades de Nussbaum). Desde estas perspectivas, se sostiene que el trato corriente que se da a los refugiados, a los desplazados medioambientales y a los inmigrantes económicos, amenaza su dignidad y debe, por tanto, dar paso a otros modos de hacer frente al fenómeno migratorio.

Palabras clave: dignidad humana; inmigración; refugiados; justicia

\footnotetext{
Proyecto Fondecy regular $\mathrm{n}^{\circ} 1160982$

2 Doctor en Filosofía na Universidade Eberhard-Karls de Tübingen. Profesor titular de Filosofía Política da Escuela de Gobierno de la Universidad Adolfo Ibáñez en Santiago de Chile. ORCID ID: http://orcid.org/0000-0003-2230-8893
} 


\title{
Resumo
}

$\mathrm{O}$ artigo analisa como se relaciona a premissa normativa da dignidade humana a partir do fenômeno migratório. Por isso, ele dá conta de uma concepção da dignidade humana que não recorre aos pressupostos naturalistas nem supraempiricos, e apresenta três alternativas diferentes para outogar-Ihe conteúdo normativo com base em um limiar suficientarista (a teoria das relações internacionais de Rawls, a sociedade decente de Margalit, e o enfoque nas capacidades de Nussbaum). Sob estas perspectivas, sustenta que o tratamento concedido aos refugiados, aos deslocados por motivos ambientais e aos migrantes econômicos, ameaça a sua dignidade e deve, portanto, dar lugar a outras maneiras de lidar com o fenômeno da migração.

Palabras chave: dignidade humana; imigração; refugiados; justiça.

\begin{abstract}
The article analyses relate to the premise with rules of human dignity with the evaluation of the phenomenon of migration. For that, it accounts for a conception of human dignity that does not resort to naturalist or supra-empirical presuppositions, and presents three different alternatives to grant normative content based on a threshold sufficientarian (the theory of international relations of Rawls, Margalit decent society, and focus on the capabilities of Nussbaum). From these perspectives, held that the current treatment accorded to refugees, environmental refugees and economic migrants, threatening their dignity and must, therefore, give way to other ways of dealing with the phenomenon of migration.
\end{abstract}

Keywords: human dignity; Immigration; refugees; Justice.

Los movimientos migratorios se han posicionado en el foco de la atención de la sociedad civil global, constituyendo un tema de urgencia política y humanitaria. Por cierto, no es la novedad del fenómeno en cuanto tal, ${ }^{3}$ ni la cantidad de personas que cambia su residencia en el contexto internacional, ${ }^{4}$ lo que explica esta urgencia. El contexto explicativo se

\footnotetext{
3 La migración humana es parte de la historia y desarrollo de la humanidad, también de su historia reciente, de lo que dan cuenta los grandes movimientos de migratorios de comienzos del siglo XX. Compare Dummett (2001).

4 Según los datos presentados por las Naciones Unidas para el año 2015 corresponde aproximadamente el $3.3 \%$ de la población global. Esta cifra es relativamente moderada. Internatio nal Migration Report 2015.
} 
relaciona con las grandes desigualdades económicas entre los países, que en muchos casos amenaza y menoscaba las condiciones de vida, y con un contexto internacional constituido por Estados nacionales que, en nombre de la soberanía, reclaman y se reconocen mutuamente un derecho de exclusión. Es así como un objetivo prioritario de la política estatal es la implementación de estrategias para evitar el acceso de inmigrantes (dejando incluso sin efecto la Convención de Refugiados de Ginebra que regla las condiciones de acceso de los refugiados). En la discusión filosófica, además de la urgencia del fenómeno, su actualidad temática se relaciona con la estructura corriente de las teorías de justicia. Por una parte, las teorías dominantes se caracterizan por un foco estado-céntrico. Por otra parte, sus propios presupuestos normativos en ocasiones implican posicionamientos normativos de alcance universal. Este puede ser el caso de las exigencias de justicia global, y de un modo central para este artículo, para la estipulación de la dignidad humana.

En este artículo analizaré cómo se relaciona la premisa normativa de la dignidad humana con la evaluación del fenómeno migratorio. Procederé en seis pasos. En primer lugar, daré cuenta de una concepción de dignidad humana que no recurre a presupuestos naturalistas ni supraempíricos. Luego presentaré tres alternativas diferentes para dar contenido normativo a la dignidad. En tercer y cuarto lugar, me referiré someramente al fenómeno migratorio en la actualidad, y examinaré cómo la referencia a la dignidad humana debiese llevar a evaluar este fenómeno. Luego me referiré al alcance de las obligaciones que se siguen del reconocimiento de la dignidad humana en el caso de la migración y los refugiados. Finalmente resumiré las conclusiones del artículo.

\section{Dignidad humana sin naturalismo y sin metafísica}

En los documentos jurídicos nacionales e internacionales la referencia a la dignidad humana es omnipresente. En el preámbulo de la Carta de las Naciones Unidas del 26 de junio de 1945 se afirma: "Nosotros los 
pueblos de las Naciones unidas [estamos] resueltos a reafirmar la fe en los derechos fundamentales del hombre, en la dignidad y el valor de la persona humana..." En el artículo primero de la Declaración general de los derechos humanos de 1948 se afirma que "Todos los seres humanos nacen libres e iguales en dignidad y derechos...". La referencia a la dignidad humana también ocupa un lugar central en muchas constituciones. Surgida como respuesta a los horrores del nazismo, la constitución alemana de 1949 afirma en su primer artículo: "La dignidad humana es intangible. Respetarla y protegerla es obligación de todo poder público". Sin embargo, a pesar de su ubiquidad, no es evidente cómo entenderla.

De un modo sistemático, se distinguen referencias a la dignidad entendida (i) como resultado o rendimiento y (ii) como propiedad humana. El primer entendimiento es común en el mundo clásico. Aquí la dignidad es un resultado obtenido ya sea por merecimientos especiales, virtud, una buena impronta, logros, o el reconocimiento de los otros. Bajo este concepto no somos todos iguales en dignidad, y la dignidad obtenida tampoco es una característica definitoria de un tipo de ser: la dignidad se puede obtener y perder. ${ }^{5}$ Pero no es este el concepto de dignidad que anima las sentencias arriba consignadas. Por el contrario, en estas la dignidad se entiende como una propiedad que pertenece a los hombres sólo por el hecho de serlo, sin referencia a ningún tipo de logro o rendimiento. Utilizando conceptos rawlsianos (que él utiliza para referir a la comunidad moral), dignidad tendría una "propiedad de rango", es decir, una propiedad que se tiene o no se tiene, sin admitir gradualidades (Rawls 1971: 508): imagine un plano sobre el que se distribuyen puntos. Al dibujar una circunferencia sobre el plano, algunos puntos quedan dentro y otros fuera. Todos aquellos que son incluidos en la clase, lo son, porque poseen la propiedad de rango. Siguiendo este entendimiento, la dignidad le corresponde a todos aquellos que están dentro de la circunferencia -en el entendimiento humanista

\footnotetext{
5 Para un instructivo estudio acerca de cómo se articulan las referencias a la dignidad en la historia de la filosofía, compare Wetz (1998, cap. 2-3)
} 
universalista prevalente, quienes pertenecen a la especie humana, también en una perspectiva diacrónica que integra a las generaciones futuras- ${ }^{6} \mathrm{Y}$ tal como no tiene sentido afirmar que un punto está más adentro de la circunferencia que otro (se puede afirmar que se está más cerca del centro de la circunferencia, pero no que se está más adentro), ya que, o se está adentro o se está afuera, tampoco tiene sentido afirmar que algunos seres humanos tienen más dignidad que otros. Por el contrario, todos tendrían dignidad y todos la tendrían en igual medida.

Pero no es evidente cómo se funda esta dignidad. ¿Se retrotrae a alguna fundación filosófica y tendría, por tanto, un estatus eminentemente moral, o es resultado de una afirmación positiva y tendría, por tanto, un estatus exclusivamente jurídico? (una discusión en Lohmann 1999). Tampoco es evidente el rendimiento del concepto: si se define por referencia a derechos fundamentales, ¿no es acaso la referencia a la dignidad superflua? Y si se define de otro modo ¿cómo definir sus implicaciones sobre un conjunto de derechos o más allá de este? No me referiré a estas discusiones.7 Sólo realizaré algunas consideraciones necesarias para construir una concepción de dignidad que resista un cierto tipo de escepticismo.

La referencia a la dignidad como propiedad naturalista humana es cuestionable. Así, por ejemplo, Peter Singer afirma que esta referencia es vacía. Ella surgiría cuando los filósofos ya no saben cómo diferenciar de un modo moralmente relevante a los seres humanos de otros animales (Singer 1993). No ahondaré en esta crítica. Pero algunas anotaciones son necesarias.

Primero: como es conocido, la referencia a la dignidad como propiedad sería vacía, porque es implausible identificar una propiedad naturalista de rango que incluya a todos los seres humanos (los que tienen dignidad) y excluya a todos aquellos que no lo son. El argumento de los seres margi-

\footnotetext{
6 Para una discusión acerca de cómo integrar a las generaciones futuras en un marco argumentativo contractual, compare Loewe (2010c).

7 Compare los artículos en Gosepath/ Lohmann (eds) (1999); y en el número Philosophie der Menschenwürde del Zeitschrifft für Menschenrechte.
} 
nales lo explicita claramente: cualquiera sea la propiedad que se proponga, dejará fuera de su rango a algunos seres humanos. A modo de ejemplo, este sería el caso de la referencia a la razón (común en círculos kantianos) o al lenguaje. No todos los seres humanos tendrían estas capacidades.

Segundo: si bien es corriente recurrir a algún argumento de potencialidad para incluirlos, estos argumentos valen en "casos normales". Pero es evidente que también hay seres humanos que jamás desarrollarán o que han perdido definitivamente estas capacidades. Así, a menos que optásemos por otra propiedad de rango que sí cumpliese la exigencia de inclusión y exclusión, estos seres humanos estarían fuera del espacio de la dignidad humana. Insistir en que la dignidad incluye a toda la especie humana denotaría simplemente un tipo de especismo, esto es, el privilegio injustificado, y por tanto ilegítimo, de ciertos seres en razón de su especie (y la correspondiente discriminación ilegítima de todos aquellos seres que no pertenecen a ella).

Tercero: un argumento más sofisticado debiese apuntar a que, aunque un ser humano nunca desarrolle esas capacidades, dado que es genéticamente humano, las podría haber desarrollado si no hubiese estado sujeto a alguna condición. Pero este argumento también criticable. Es misterioso entender porque el que un ser $X$, bajo otras circunstancias, pudo haber llegado a ser $Y$, es un argumento para tratar a $X$ como si fuese $Y$. $X$ no es $Y$-y el que en un mundo posible pudiese haberlo sido, no es $y$ no puede ser un argumento para tratarlo como lo que no es. Incluso si el argumento criticado (que el ser genéticamente humano las podría haber desarrollado) fuese plausible, de este se sigue nuevamente una consecuencia implausible: imagine que exista un desarrollo tecnológico que permita que los animales sean modificados genéticamente, de modo que adquieran las capacidades requeridas según algún concepto de dignidad. ¿Sería la mera existencia de este desarrollo un argumento para otorgar dignidad a los animales? ${ }^{8}$ Aunque se sostenga la plausibilidad normativa

8 Esta es una modificación del argumento desarrollado por Talbert (2006). 
de la afirmación de la dignidad animal -como yo lo hago-, ${ }^{9}$ sería al menos curioso sostenerla en base a este argumento.

Una opción alternativa es desechar propiedades naturalistas y defender, en su lugar, algún tipo de propiedad supraempírica. Esta es la suposición corriente que respalda la referencia a la dignidad en la actualidad como propiedad. La imagen y semejanza del hombre con Dios, la posesión de un alma inmortal, o la razón entendida como una condición a priori, han sido y son candidatos usuales. Pero si bien estos candidatos pueden establecer una propiedad de rango que cumple la condición de inclusión y exclusión expuesta, no son aceptables sin más. Esto se debe a que recurren a peticiones de principio que no son aceptables en sociedades contemporáneas, no al menos, sin cualificaciones importantes.

Lo dicho se puede explicitar por referencia al liberalismo político rawlsiano y su reconocimiento del "factum del pluralismo" para denotar la pluralidad de concepciones morales como una característica central e inevitable de las sociedades liberales contemporáneas (Rawls 1993a). Rawls utiliza el concepto de "doctrinas comprehensivas" para referir a aquellos sistemas de creencias morales que especifican no sólo las instituciones políticas, sino un amplio rango de asuntos, como aquello que constituye una vida valiosa, las virtudes a desarrollar, etc. Doctrinas comprehensivas incluyen usualmente posicionamientos metafísicos y epistemológicos acerca de creencias morales. Rawls refiere a doctrinas comprensivas religiosas, morales y filosóficas. A modo de ejemplo refiere a las religiones, al kantismo y al utilitarismo. Dado que en las sociedades liberales contemporáneas hay desacuerdo en muchos asuntos que remiten a doctrinas comprehensivas, discusiones públicas acerca de asuntos constitucionales fundamentales y asuntos de justicia básica ${ }^{10}$ no deben desarrollarse ni

\footnotetext{
9 Compare Loewe (2011).

10 Los asuntos constitucionales fundamentales refieren a los principios generales que especifican la estructura general del gobierno y el proceso político, los poderes del ejecutivo, el legislativo y el judicial, el área de aplicabilidad de la regla de mayoría, y a los derechos y libertades básicas iguales de la ciudadanía que las mayorías legislativas tienen que respetar, tal como el derecho
} 
definirse por referencia a doctrinas comprehensivas. Afirmar lo contrario implica no respetar a todos aquellos que no las comparten, esto es una forma de violencia que, a su vez, amenaza la estabilidad social.

El desacuerdo en estas sociedades no es meramente contingente. Cuando los individuos pueden hacer uso de su libertad, obtienen conclusiones diversas acerca de lo valioso. Con los conceptos de Rawls, individuos razonables reconocen los "límites del juicio". Estos límites incluyen evidencia contestada, ponderaciones diferentes, indeterminación conceptual, experiencias diferentes y conflictos de valores. Por una parte, el pluralismo se sigue de los límites del juicio: en tanto los individuos sean razonables, y así reconozcan estos límites, no tienen razones para no tolerar los puntos de vista de los otros e imponer los propios. Pero, por otra parte, este mismo pluralismo torna inviable recurrir a las doctrinas comprehensivas, ya sean morales, religiosas o filosóficas, como legitimadoras del orden institucional, al menos en cuestiones constitucionales y de justicia básica. Si esto es así ¿cómo podríamos recurrir a propiedades supraempíricas -la semejanza con Dios, el alma inmortal, la razón a priori, etc.- para justificar alguna concepción de dignidad humana? Si la dignidad humana se basa en la aceptación de una doctrina comprehensiva, entonces la apelación a ella es inapropiada en sociedades plurales contemporáneas.

Pero si la dignidad humana no se funda ni en propiedades empíricas ni supraempíricas, ¿no hay acaso dignidad? Y si la hay ¿en qué se funda? La posición que aquí defiendo es que, sin naturalismo ni metafísica, la dignidad debe ser entendida como una tarea humana de conformación o creación: la dignidad humana sería el resultado del reconocimiento y respeto mutuo de los seres humanos." Así entendida, la dignidad humana

\footnotetext{
a voto y a participar en política, libertad de consciencia, de pensamiento y de asociación, y el imperio del derecho. Los asuntos de justicia básica refieren a consideraciones distributivas (Rawls 1993a: 227). En asuntos que no expresan asuntos constitucionales fundamentales o cuestiones de justicia básica los oficiales políticos y los ciudadanos pueden apelar a sus doctrinas comprehensivas.
}

"Una posición similar a la defendida defendida por Jeremy Waldron (2014; 2015). 
se realiza, cuando los hombres interactúan con respeto mutuo, lo que implica decencia, consideración de los intereses de los otros, y por cierto, y de un modo fundamental, el reconocimiento recíproco como personas con iguales derechos. Con las palabras de Tugendhat: "en la medida en que respetamos a un ser humano como sujeto de derecho, frente al cual tenemos deberes absolutos, le otorgamos dignidad y un valor absoluto. Así, de ese modo, se define el valor absoluto y la dignidad, y no como algo presupuesto dado" (1995: 145; Trad. DL). Desde esta perspectiva, no es la dignidad humana -ya sea como propiedad naturalista o supraempírica- la que está a la base del respeto mutuo y reconocimiento recíproco, sino que, inversamente, la dignidad se sigue del respeto mutuo y el reconocimiento recíproco. ${ }^{2}$ De este modo, la dignidad es una realización permanente de la acción humana. Y tal como esta acción, y las instituciones que la regulan y coordinan, pueden realizarla, cuando el reconocimiento y respeto mutuo no se dan, también pueden destruirla. Así, adquiere sentido afirmar que la dignidad se puede obtener, pero también perder, dependiendo de las condiciones de reconocimiento recíproco y respeto mutuo.

En un sentido, este entendimiento de la dignidad humana se asemeja al modo clásico de entender la dignidad ya examinado (como resultado o como rendimiento). Pero se diferencia de aquella en modos importantes. En primer lugar, no son logros o resultados singulares de los individuos los que al ser reconocidos por los otros les otorgan dignidad, y cuya ausencia implica su pérdida. Sino que se trata de un derecho que nos corresponde en la medida que nos reconozcamos como sujetos de respeto y reconocimiento mutuo. Así, podemos perder la dignidad cuando las condiciones de vida son indignas (esto es, cuando estas no corresponden al respeto y reconocimiento mutuo), pero no por esto perdemos el derecho a una vida en condiciones dignas. Al ser resultado de condiciones de recono-

12 Esta posición (la dignidad humana no es precondición del respeto humano, sino su resultado) es defendida por Tugendhat (1995) y, caracterizada de un modo diferente, también por Niklas Luhmann (1965). 
cimiento y respeto mutuo, ella es siempre exigible. Incluso, es frente a condiciones de indignidad cuando más notamos la importancia y urgencia de salvaguardar la dignidad humana (Wetz 1998: cap. 5). En segundo lugar, esta demanda por condiciones de vida dignas no es limitable a clases de individuos particulares, ya sea en razón de su mérito, su raza, nacionalidad, religión o cualquier otro tipo de adscripción particular. Tampoco lo es en razón de la ciudadanía. Si bien de la ciudadanía compartida se pueden desprender obligaciones especiales (compare Loewe 2010b), su ausencia no implica la inexistencia de obligaciones. La demanda por dignidad permanece, incluso en ausencia de consideraciones normativas relacionales. Es en este sentido que esta concepción de dignidad es eminentemente moderna y cosmopolita: la demanda por dignidad corresponde a todos los seres humanos, y a todos en igual medida.

\section{2. ¿Dignidad para qué?}

He dado cuenta de la dignidad humana como resultado de una tarea permanente de realización mediante el respeto y el reconocimiento mutuo. ¿Pero qué implica este respeto y reconocimiento, y cuáles son los derechos y obligaciones que de ellos se siguen? Una respuesta plausible debe partir del reconocimiento y aceptación de la condición de vulnerabilidad de la existencia humana. Dado el tipo de seres que somos, dependemos de ciertas condiciones básicas para poder realizarnos en el mundo. Una concepción de dignidad debe relacionarse con estas condiciones -entendidas de modo objetivo. El respeto y reconocimiento mutuo debe, por tanto, dar cuenta de estas condiciones. Por una parte, este entendimiento de la dignidad, si bien se relaciona con condiciones empíricas, no descansa en ningún tipo de falacia naturalista: el peso normativo recae en el reconocimiento mutuo, no en las características propias de los seres humanos. Por otra parte, este entendimiento no es, en principio, privativo de los seres humanos: mediante esta estructura argumentativa, y bajo el supuesto de que disponemos de buenas 
razones para querer justificar nuestras acciones frente a los otros en base a principios que podemos esperar que los otros razonablemente no puedan rechazar, es posible extenderla a ciertos animales. ${ }^{13}$

Este entendimiento de la dignidad admite interpretaciones con alto grado de variabilidad en cuanto a las exigencias que conlleva. En lo que sigue no elaboraré una concepción substantiva de dignidad, sino que sólo esbozaré algunas posibilidades, sin posicionarme críticamente frente a ellas.

En el contexto de las sociedades liberales, Rawls recurre al reconocimiento de la libertad e igualdad de los ciudadanos, y en base a un entendimiento de la sociedad como empresa cooperativa, a la exigencia del reconocimiento de la reciprocidad, entendida como la motivación para justificar las propias acciones frente a los libres e iguales mediante principios que razonablemente podamos esperar que los otros puedan aceptar (Rawls 1999). Esta es la condición de razonabilidad. Según Rawls, de este reconocimiento se sigue la aceptación de ciertos principios políticos. En último término, se debe reconocer tres principios: uno que establece libertades fundamentales; uno que establece la prioridad de este principio por sobre consideraciones del bien común; y uno que establece un mínimo material como necesario para hacer uso de las libertades. Según el entendimiento defendido en este capítulo, la dignidad se realizaría mediante el reconocimiento de alguna interpretación de los tres principios enunciados. Pero a pesar de la su atractividad sustantiva (o por ella misma), esta interpretación estipulativa del contenido de la dignidad es demasiado exigente, ya que privilegia sobremanera sociedades liberales por sobre otro tipo de sociedades que, sin ser liberales, aún podrían tener una concepción de dignidad humana razonable.

Una interpretación suficientarista parece ser más apropiada, al menos si se aspira a extender la concepción de dignidad más allá de aquellas

13 En otro sitio he argumentado en este sentido, en base a la teoría contractualista de Thomas Scanlon (Loewe 2016). 
fronteras políticas dentro de las que priman las instituciones liberales. ${ }^{14}$ Considere la posición de Margalit en La Sociedad Decente (1997). Si bien Margalit argumenta a favor de cambiar la búsqueda de la dignidad humana por la pregunta acerca de por qué es incorrecto humillar a los seres humanos, los modos de humillación que identifica se pueden entender como correlatos de una concepción de dignidad suficientarista como la que aquí he defendido, una que se basa en el respeto y reconocimiento mutuo. Margalit identifica tres modos de humillación: tratar a los seres humanos como subhumanos, como máquinas, o como ciudadanos de segunda clase; privación del autocontrol mediante un poder totalitario; y exclusión de determinadas personas o grupos de personas de la comunidad humana. Una sociedad respeta la dignidad cuando los ciudadanos y sus instituciones no ofrecen razones a los individuos para que puedan considerar que su auto-respeto está siendo violado.

El mismo Rawls, al extender su teoría al Derecho de Gentes, no recurre a los tres principios liberales enunciados en su teoría doméstica de la justicia, sino que a la "decencia" de las sociedades: esta es la condición que los pueblos deben cumplir para poder ser considerados como miembros iguales con los mismos derechos del Derecho de Gentes. Sociedades no liberales, pero que pueden ser consideradas decentes, son miembros de la sociedad de pueblos. ${ }^{15} \mathrm{~A}$ diferencia de las condiciones impuestas al nivel de justicia doméstica, las condiciones de decencia son menos exigentes. Sociedades jerárquicas decentes -como él las denomina-se definen mediante tres características (Rawls 1999: 64-5): (1) no son agresivas; (2) se organizan mediante una concepción común del bien -que incluye un sistema de consultación; (3) y respetan los derechos humanos. Ya la segunda condición deja en claro que las sociedades decentes no pueden ser

\footnotetext{
14 Tal posición que se puede suponer a la base de las palabras de Schiller: "Würde des Menschen - Nichts mehr davon, ich bitt euch. Zu essen gebt ihm, zu wohnen, habt ihr die blosse bedeck, gibt sich die Würde von selbst" (Schiller 1962: 248)

15 En otro lugar he discutido el Derecho de Gentes rawlsianos y el rol de las sociedades jerárquicas decentes en esta teoría (Loewe 2015a).
} 
liberales -estas últimas no pueden organizarse mediante una concepción del bien. El respeto de los derechos humanos es una condición necesaria (aunque no suficiente) para la decencia de las instituciones políticas y sociales domésticas (Rawls 1999: 80). Rawls sostiene una concepción minimalista de derechos humanos, según la cual no todos los derechos fundamentales pertenecen a la clase de los derechos humanos. Los derechos humanos "expresan una clase especial de derechos urgentes" (Rawls 1999: 79). A esta clase pertenecen: (a) el derecho a la vida (a aquello que es necesario para la propia subsistencia y seguridad); (b) el derecho a la libertad (ser libre de esclavitud, de trabajo forzado, una medida suficiente de libertad de conciencia que garantice la libertad de religión y pensamiento); (c) el derecho a propiedad personal; $y$ (d) el derecho a igualdad formal (casos iguales deben ser tratados de igual modo) (Rawls 1999: 65). También refiere a la protección de grupos étnicos contra genocidio (Rawls 1999: 79). ${ }^{16}$ Si aplicamos la concepción de dignidad desarrollada a esta concepción, podemos afirmar que el correlato de la dignidad es el respeto de los derechos humanos, entendidos de un modo minimalista, que Rawls considera condición necesaria -aunque no suficiente- para ser parte del Derecho de Gentes.

Una tercera estrategia para dar contenido a la dignidad humana la encontramos en el enfoque en las capacidades, como ha sido articulado y defendido especialmente por Martha Nussbaum. Central para el enfoque (también en la versión de Sen), es que la métrica de una teoría igualitaria debiese constituirse en base a capacidades, y no a medidas subjetivas del bienestar, o a bienes objetivos -tales como recursos o bienes primarios - sin referencia a lo que los seres humanos pueden hacer con estos bienes (Loewe 2009). Dado que las tasas de

\footnotetext{
16 Las demandas normativas que Rawls reconoce como derechos humanos están bastante más atrás de aquello que en los documentos internacionales directrices se reconoce como derechos humanos. Entre estos últimos se encuentran derechos como la libertad de opinión, de expresión y de prensa; la libertad de reunión y de asociación; el derecho a participación política, a educación y a atención en salud.
} 
convertibilidad de bienes en ventajas varían entre los individuos en modos que no se retrotraen a su responsabilidad ${ }^{17}$, la justicia debiese enfocarse en lo que los seres humanos pueden llegar a ser y hacer con los bienes. Según la interpretación de libertad razonada desarrollada por Sen, las capacidades se deben entender como libertades substantivas, para elegir la vida que uno tiene razones para valorar (Sen 1999: 74). En la interpretación de Nussbaum, que es la que aquí me interesa, las capacidades se definen y especifican en razón de que posibilitan ciertos tipos de funcionamiento considerados especialmente valiosos, porque posibilitan una vida con dignidad humana. ${ }^{18}$

Nussbaum diferencia entre tres diferentes tipos de capacidades (Nussbaum 2000: 84 y siguientes). Las capacidades básicas refieren al equipamiento innato de los seres humanos -por ejemplo, la capacidad de ver. En base a estas se pueden desarrollar capacidades más avanzadas. Las capacidades internas refieren a condiciones maduras, es decir, estados desarrollados que son condición para poder ejercer los funcionamientos. En ocasiones estas capacidades requieren de tiempo para madurar (por ejemplo, la capacidad de tener funcionamiento sexual) bajo ciertas condiciones (por ejemplo, alimentación). En otras, requieren exposición a ciertos tipos de actividad. Es el caso del aprendizaje de un lenguaje en el caso de los niños. Están también las capacidades combinadas. Aunque se hayan desarrollado las capacidades básicas y las internas, es posible que haya un impedimento para el funcionamiento relacionado. Es el caso, por ejemplo, de una mujer que ha desarrollado la capacidad interna del funcionamiento sexual, pero no puede ejercerla ya que ha enviudado y vive en una sociedad tradicional que no permite a las mujeres casarse nuevamente (Nussbaum 2000: 85).

\footnotetext{
17 Sen refiere a (i) heterogeneidades personales; (ii) diversidades medioambientales; (iii) variaciones en el clima social; y (iv) diferencia en expectativas relacionales. (Sen 1999: 70-71) 18 Nussbaum amplia esta concepción para dar cuenta de la dignidad animal en base a la determinación de los funcionamientos propios de la especie (Nussbaum 2006).
} 
Así, las capacidades combinadas refieren a la interacción entre capacidades internas y el medioambiente institucional, social y cultural.

Nussbaum enumera una lista de diez capacidades combinadas centrales para poder desarrollar una vida con dignidad humana. Esta lista incluye (1) vida -ser capaz de vivir una vida de duración normal; (2) salud física, incluyendo salud reproductiva, alimentación y habitación adecuada; (3) integridad corporal; (4) sentidos, imaginación y pensamiento; (5) emociones; (6) razón práctica; (7) afiliación, lo cual implica no sólo ser capaz de vivir con y hacia otros, sino también las bases sociales del autorespeto; (8) relación hacia otras especies; (9) juego; (10) control sobre el medioambiente, tanto político como material (Nussbaum 2000: 78-80; la lista es reconsiderada en 2006: 76-78). La filiación y la razón práctica son especialmente importantes. Sin embargo, según Nussbaum, todas las capacidades combinadas son relevantes, ya que posibilitarían el florecimiento humano. A partir de un determinado nivel, su posesión puede hacer posible una buena vida. Pero incluso bajo este nivel, es importante disponer de ellas por sobre un umbral suficientarista. Es este umbral suficientarista el que definiría la dignidad humana.

\section{Inmigrantes y refugiados}

Según los datos de las Naciones Unidas, ${ }^{19}$ el año 2015 la cantidad de migrantes internacionales, entendiendo por tales aquellos que viven en un país distinto al de su nacimiento, alcanzó 244 millones. Si bien la cifra muestra un aumento de $41 \%$ con respecto a la del año 2000 , ella continúa siendo relativamente moderada (3.3\% de la población global). Considerando la distribución regional, migrantes internacionales así definidos, constituyen aproximadamente el 10\% de la población de Europa, Norteamérica y Oceanía, mientras que menos del $2 \%$ en África, Asia, y América Latina y el Caribe. Las causas de la migración son diversas. Es

19 International Migration Report 2015. 
común diferenciar entre pull-factores (los que atraen al inmigrante) y push-factores (los que empujan al emigrante a abandonar su país de origen), y relacionar los primeros con los inmigrantes económicos y los segundos con los refugiados. Pero en muchos casos ambos factores actúan simultáneamente: la atracción de un destino es una función de la del lugar de origen, y viceversa.

Entre las causas de la inmigración se encuentran razones relativas a la consecución del propio plan de vida, la búsqueda de más y mejores oportunidades, la huida de condiciones miserables de vida, de medioambientes degradados, de persecución y guerras, de violación de derechos fundamentales, de devastación, y la amenaza vital inminente. Según datos de la UNHCR, el año 2015 había más de 65 millones de desplazados forzados. Por cierto, la mayor cantidad buscan refugio dentro de las fronteras del propio país. Pero entre los desplazados forzados la UNHCR cifra en más de 21 millones la cantidad de refugiados internacionales, siendo más de la mitad menores de edad. La mayor cantidad de refugiados proviene de 3 países: Somalia (1.1 millones), Afganistán (2.7 millones), y Siria (4.9 millones). ${ }^{20}$ Alcanzando cifras record, la UNHCR proyecta para el año 2017 en 1.19 millones la cantidad de personas en situación de necesidad en búsqueda de reasentamiento, cifra que supera por mucho las oportunidades disponibles en países terceros. Las rutas de los inmigrantes y refugiados suelen ser peligrosas. Según cifras de UNHCR el año 2016 se alcanzó el record de 5022 muertos en la ruta del Mediterráneo a Europa (el 2015, la estimación fue de 3771). Organizaciones de ayuda estiman que la cifra podría ser mayor. No sólo la cifra es enorme, lo es también la probabilidad de morir en el intento de alcanzar las costas de Europa: según Médicos sin Fronteras, el año 2016 murió una de cada 41 personas que intentó alcanzar Italia. Cerca del $16 \%$ de los que alcanzan las costas italianas son niños, la mayoría sin acompañamiento.

20 Contrariamente a lo que sugiere la prensa, los países que más refugiados cobijan son Jordania, Etiopía, Irán, el Líbano, Paquistán y Turquía. 
Si bien algunos países han realizado esfuerzos para acoger refugiados que huyen de conflictos armados, esta no es la regla. El exjefe del alto comisionado de derechos humanos de las Naciones Unidas, Zeid Ra'ad al-Hussein, criticó en numerosas ocasiones la "indiferencia" que los líderes europeos muestran hacia la muerte de miles de refugiados que intentan huir: "muchas personas comunes y corrientes en Europa han dado la bienvenida y apoyado a los migrantes, pero los líderes políticos muestran una indiferencia escalofriante hacia su destino". ${ }^{21}$ Zeid Ra'ad al-Hussein apuntó especialmente a las narrativas escogidas por los políticos para deliberadamente generar miedo y pánico en la población, transformando a personas vulnerables en hordas criminales invasoras. Esto no refiere sólo a retóricas populistas y nacionalistas. El Gobierno británico terminó un programa de reasentamiento de niños refugiados no acompañados. Hungría construye una valla para mantener fuera a los migrantes. La Unión Europea utiliza a la milicia libia para retener a los refugiados en Libia, un país devastado por la guerra. La administración Trump aspira a impedir el ingreso de refugiados e inmigrantes de una serie de países mayoritariamente musulmanes, entre los que se cuentan países en conflicto, así como a impedir el ingreso de refugiados e inmigrantes mediante la construcción de un muro. Incluso Alemania, quién de un modo excepcional en el concierto internacional, ha recibido grandes cantidades de refugiados, se presta a retornarlos tan pronto como las descripciones formales permiten estipular que se trata de sitios seguros, lo que no siempre es el caso.22

Si la dignidad es resultado del respeto y reconocimiento que nos debemos, la pregunta es si el trato corriente a los inmigrantes y refugiados está acorde con aquella. En el derecho internacional, la emigración es reconocida como un derecho humano (Artículo 13-2 de la Declaración

\footnotetext{
${ }^{21}$ Disponible en: http://www.independent.co.uk/news/world/europe/refugee-crisis-migrants-asylum-seekers-latest-un-zeid-hussein-human-rights-chilling-indifference-a7619301.html

22 A modo de ilustración: mientras escribía estas líneas estaba programado el retorno de refugiados a Kabul por considerarla una zona segura. Ese mismo día una bomba mató a más de 150 personas en esa ciudad -lo que aplazó su retorno.
} 
Universal de Derechos Humanos). Pero este derecho humano no implica un derecho a la inmigración o una obligación de parte de otras naciones a las de origen, a aceptar el ingreso de estos emigrantes. Es decir, hay un derecho humano a emigrar, pero no hay un derecho humano a inmigrar. Sin duda, aun cuando esta asimetría tiene sentido, es cuestionable el valor de un derecho a emigrar sin un derecho correspondiente a no ser expulsado en las fronteras, sobre todo en un mundo de fronteras cerradas. Por su parte, el caso de los refugiados es reglado por la convención de Refugiados de Ginebra, que surgió de los grandes movimientos de población y desplazados que siguieron a la segunda guerra mundial. El concepto de refugiado refiere a toda persona que "debido a un temor fundado de persecución en razón de su raza, religión, nacionalidad, pertenencia a un grupo social particular o en razón de su convicción política, se encuentra fuera del país cuya nacionalidad posee, y que no puede demandar la protección de éste o en razón de esos temores fundados no la quiere demandar" (art. 1, A.1). El artículo 33.1 constituye el núcleo de la Convención, estableciendo la prohibición de expulsión (la condición de non-refoulment). De acuerdo a esta: "ninguno de los Estados partes de este contrato expulsará de algún modo a un refugiado sobre las fronteras de los territorios en los que su vida o su libertad, en razón de su raza, religión, ciudadanía, su pertenencia a un grupo social particular o en razón de su convicción política, fuese amenazada." La Convención ha encontrado sólo parcial aceptación en el derecho interno de los países firmantes. Esto se debe a que -como en muchos otros convenios del derecho internacional- falta un instrumento para convertirlo en derecho vinculante (ius cogens). Usualmente los Estados consideran el modo de tratar la protección de los refugiados como un ámbito de la política sobre el que pueden disponer arbitrariamente. A esto se suma que las disposiciones más importantes de la Convención están formuladas como obligaciones de los Estados y no como derechos subjetivos de los refugiados que, en cuanto tales, se podrían reclamar. 


\section{La dignidad del inmigrante y del refugiado}

¿Por qué migran las personas? Las respuestas son variadas, pero en todas ellas hay un común denominador: "people move in the hope of a better life" 23

Considere a los inmigrantes. En el debate se suele considerar que en su caso priman los pull-factores, es decir, son motivados por la atractividad del lugar de destino. Por tanto, se subentiende que el proceso de migración es voluntario. No estarían compelidos a abandonar el lugar de origen, sino que deciden hacerlo en base a sus expectativas. Por ejemplo, en su defensa de derechos culturales de minorías, Will Kymlicka (1995) establece una diferenciación entre grupos nacionales e inmigrantes. La distinción juega un papel central en su teoría porque, según ésta, son las minorías nacionales las que legítimamente pueden demandar derechos de autogobierno, pero no los inmigrantes. Estos últimos sólo pueden reclamar derechos poliétnicos, entendidos como derechos que facilitan la integración en la cultura societal receptora. Según Kymlicka, la voluntariedad de la inmigración implica que al abandonar su patria los emigrantes pierden sus derechos nacionales. A la base de esta distinción está una idea central del liberalismo igualitario: hay cosas por las que no podemos considerar a los individuos como responsables -las condiciones socieconómicas en las que fuimos socializados, nuestros talentos naturales, etc.-, pero otras por las que sí, eminentemente nuestras decisiones. Por lo tanto, es razonable esperar que cada cual se haga responsable de los costos que se siguen de sus propias decisiones voluntarias, pero no de todos los costos que se retrotraen a circunstancias que nos desaventajan sin haber sido nunca objeto de nuestra decisión (una discusión y bibliografía en Loewe 2008). Esta es la razón a la base de la afirmación de que los inmigrantes no pueden reclamar legítimamente en el país de destino aquellos derechos de

\footnotetext{
23 "Why do people move? What makes them uproot and leave everything they've known for a great unknown beyond the horizon? Why climb this Mount Everest of formalities that makes you feel like a beggar? Why enter this jungle of foreignness where everything is new, strange and difficult? The answer is the same the world over: people move in the hope of a better life." Life of Pi (Martel 2001).
} 
autogobierno que les corresponde a los grupos nacionales. Sin embargo, esta tesis no se sostiene sin cualificaciones importantes: la inmigración no es siempre voluntaria.

Un indicio de la falta de voluntariedad se presenta cuando los costos de no emigrar son muy altos. Ciertamente, los costos que la inmigración implica para los inmigrantes son altos. La inmigración es un proceso doloroso (Vitale 2006). Incluso, como Kymlicka admite, la integración social en el país de acogida no es siempre posible, debido a causas culturales idiosincráticas. Pero a pesar de estos altos costos, en ocasiones los costos asociados a la permanencia son incluso mayores. Sin duda, esto aún no es conclusivo para determinar la ausencia de voluntariedad en el caso de la migración. Pero cuando la permanencia en el lugar de origen va asociada a condiciones de vida deplorables, al menos por referencia a algún umbral medianamente objetivo, la idea de que la motivación del migrante se retrotrae a los pull-factores pierde en plausibilidad explicativa: en estos casos, perece ganar plausibilidad la idea de que los push-factores son determinantes. Y si es así, entonces pierde fuerza la idea de que la migración -en estos casos limitados- es voluntaria, es decir, expresión de la voluntad no constreñida de modos inaceptables. Pero si esto es así, se sigue que no es razonable estipular que el inmigrante potencial deba cargar con todos los costos de su proceso de migración, por ejemplo y entre otros, con aquellos que se siguen del hecho de ser rechazado, o con una travesía insegura y en demasiadas ocasiones letal, o con condiciones de acogida deplorables. Ciertamente, como anuncié al comienzo, en la mayoría de los casos, aunque no en todos, los pull y push-factores actúan en conjunto: la atractividad de un destino se relaciona con las condiciones del origen ( $y$ la evaluación de las condiciones de origen con la atractividad de un destino). Pero en la medida que reconocemos un umbral mínimo como necesario para una vida en condiciones aceptables, gana plausibilidad la idea de que la decisión de emigrar de aquellos que están por debajo de ese umbral, no es más voluntaria que la de aquel que se decide por un curso de acción en razón de que toda otra opción sería 
intolerable (como la de aquel que entrega su bolsa al asaltante, porque la opción alternativa es entregarle la vida). La tesis aquí defendida, es que este umbral está definido mediante las condiciones que hacen posible hablar de una vida humana digna. El proceso de emigración de aquellos que en su país de origen están por debajo de este umbral, es uno que se puede caracterizar como una búsqueda de dignidad. $Y$ el reconocimiento de una demanda legítima a inmigrar en todos estos casos, corresponde al reconocimiento de su universalidad.

Según la conceptualización realizada, la dignidad humana se sigue del respeto mutuo y reconocimiento recíproco. En razón de la vulnerabilidad humana, la dignidad se relaciona con condiciones mínimas que permiten un cierto desarrollo humano. He argumentado a favor de una concepción umbralista suficientarista. Pero incluso condiciones mínimas corrientemente no se cumplen en el caso de los inmigrantes y refugiados. En el caso de estos últimos -entendidos en el sentido limitado estipulado por la Convención de Refugiados de Ginebra, es decir, como aquellos que huyen de persecución-, esto resulta evidente. Las condiciones de persecución que los impelen abandonar su país de origen, los colocan por debajo de este umbral suficientarista. Según la Convención, para ser considerados como tales, los refugiados se deben encontrar fuera del país de su nacionalidad. Pero resulta evidente que las circunstancias que menoscaban sus condiciones de vida se dan, en primer lugar, dentro del país de origen. En segundo lugar, se dan en los procesos mediante los cuales tratan de alcanzar un destino seguro. Como afirmé, las rutas del Mediterráneo tienen un triste record de muerte: se estima que el año 2016 murieron 5022 en ellas (uno de cada 41 personas que intento alcanzar las costas italianas). Y las interminables filas de refugiados agolpados en las fronteras cerradas tornan patente la vulnerabilidad humana. En tercer lugar, están las condiciones de vida de los refugiados en los países de destino mientras se tramitan sus solicitudes. Estas varían bastante. Pero en uno de sus extremos, aquel caracterizado por campos de refugiados y 
por internación en sistemas de régimen de tipo cuasi carcelario, se trata de condiciones de vida que menoscaban la dignidad humana.

Pero no es sólo la dignidad de los refugiados (en el sentido de la Convención), la que está siendo amenazada. Como examinamos, otros inmigrantes, que no están siendo perseguidos, también huyen, en un cierto sentido, por su vida. Considere el caso de los inmigrantes medioambientales. Es un hecho reconocido por la comunidad científica que una de las mayores causas de la migración en el futuro cercano se relacionará con las consecuencias del cambio climático. Las estimaciones de desplazados medioambientales para el año 2050 varían entre 50 y 1000 millones. La cifra de 200 millones presentada por Myers (2005) ha llegado a ser ampliamente aceptada, siendo recogida en estudios como el Stern Review (2006) y en las publicaciones del IPCC. Entre la degradación medioambiental y la migración se producen múltiples relaciones. El ejemplo paradigmático de una relación causal directa entre el cambio climático y la migración se da, a modo de ejemplo, en el caso de los Estados Islas del Pacífico (Minura 2007; compare Brown 2011: cap.6). Por ejemplo, Kiribati, un Estado compuesto por islas que apenas se elevan sobre el nivel del mar, está amenazado por las inclemencias del tiempo, el aumento del nivel del mar y la salinización del agua y la tierra, lo que las tornará inhabitables. 0 Tuvalu, cuyos líderes ya han anunciado que abandonarán la isla. Otro tipo de relación se da cuando el cambio climático es uno entre otros factores en la explicación de la migración: "Migrants respond to economic, social, and demographic factors in addition to the environment. Assessing environmental influences on migration is complex and must take these other factors into account" (Gray 2010: 6) La capacidad para hacer frente al riesgo del cambio climático depende no sólo de la disposición sino también de posibilidades materiales. Correspondientemente, no hay distribución equitativa de las consecuencias negativas del cambio climático, debiendo cargar corrientemente los más pobres - sea por falta de recursos o administración ineficiente-con más y peores consecuencias negativas, ya que disponen de menos oportunidades para implementar medidas de 
mitigación y adaptación. Es así como el cambio climático actúa como uno más entre otros factores en el desplazamiento, aunque es difícil estipular como se relacionan estos factores. La degradación medioambiental y la falta de oportunidades que la acompañan impelen a muchos a migrar para buscar mejores oportunidades económicas, pero estos son usualmente denominados inmigrantes económicos. Sea como sea, es evidente que la degradación ambiental juega un papel, al menos uno entre otros, en el desplazamiento involuntario. Si esto es así, es razonable considerar que los desplazados medioambientales, y los migrantes económicos que huyen de la falta de oportunidades que trae consigo la degradación ambiental y que amenaza su dignidad, son refugiados climáticos (Loewe 2014).$^{24}$ Si la situación de los refugiados climáticos o económicos en razón de la degradación medioambiental es tal, que están por debajo del umbral suficientarista estipulado, entonces su dignidad está siendo amenazada.

Pero la amenaza a la dignidad excede a los perseguidos y los desplazados medioambientales. En buena medida, las causas de la migración refieren a la miseria y falta de oportunidades que suelen acompañar a la pobreza. Vivimos en un mundo caracterizado por grandes desigualdades. En términos del Coeficiente de Gini, la desigualdad global es mayor que la desigualdad dentro de cualquier país individual. Según Branko Milanovic (2016), economista del Banco Mundial, esto se debe a que hoy la mayor diferencia de ingreso entre los individuos se puede atribuir al país del que uno es ciudadano, en contraste con el siglo XIX en que era atribuible a

\footnotetext{
24 Este concepto comienza a ser utilizado en las ciencias sociales. Pero es rechazado por el derecho internacional. Así ha quedado recientemente establecido por una Corte de Justicia neozelandesa que rechazó la demanda del ciudadano de Kiribati, loane Teitiotas, para ser reconocido como el primer refugiado climático. La definición usual de "refugiado medioambiental" proviene de informe de El-Hinnawi del programa de medioambiente de las Naciones Unidas: "Environmental refugees are defined as those people who have been forced to leave their traditional habitat, temporary or permanently, because of a marked environmental disruption (natural and/or triggered by people) that jeopardized their existence and/or seriously affected the quality of their life. By 'environmental disruption' in this definition is meant any physical, chemical and/or biological changes in the ecosystem (or the resource base) that render it, temporarily or permanently, unsuitable to support human life" (Elhinnawi 1985: 4).
} 
la posición que cada cual ocupaba dentro de la propia sociedad. En una medida importante, son estas desigualdades las que están a la base de los flujos migratorios. Rechazar inmigrantes económicos sin más, implica en muchos casos sostener que sus oportunidades limitadas para desarrollar una vida humana son aceptables. En muchas ocasiones, este puede ser el caso. Pero no en todas. Hay situaciones caracterizadas por conjuntos de oportunidades tan limitados, que se encuentran por debajo del umbral suficientarista que se relaciona con la dignidad humana. Cuando es imposible satisfacer necesidades básicas, tiene mucho sentido referir al concepto los refugiados económicos.

Ahora podemos considerar las tres opciones que he examinado para dar contenido a la dignidad humana: la no humillación de Margalit, la concepción minimalista de derechos humanos defendida por Rawls, y el enfoque de las capacidades en la versión de Nussbaum. Si la dignidad humana implica no humillación, entendiendo humillación como el trato a los seres humanos como subhumanos, como máquinas, o como ciudadanos de segunda clase, la privación del autocontrol mediante un poder totalitario, y la exclusión de determinadas personas o grupos de personas de la comunidad humana, resulta meridianamente claro que la situación en que se encuentran los refugiados es una caracterizada por la humillación. En muchos casos huyen de conflictos, guerras o regímenes injustos, en los que su daño es considerado como un objetivo, o como un efecto colateral aceptable. En ambos casos o se los excluye de la comunidad humana, o se los considera como subhumanos -sacrificables por fines superiores-, o al menos como ciudadanos de segunda clase. Tampoco tienen control sobre sus vidas. Esta situación se reproduce en las rutas, y en ocasiones, también en los países de acogida durante los procedimientos para obtener el estatuto de refugiado. Por su parte, los refugiados medioambientales, si bien dentro de su país no son necesariamente humillados por las instituciones, en los casos en que sus demandas por desplazamiento son negadas por otros Estados aun cuando hay una amenaza vital -sería el caso de muchas Islas Estados-, son tratados 
como subhumanos por todos aquellos Estados que estando en posición de ayudar, no lo hacen. Algo similar se puede afirmar de los inmigrantes económicos en situación de necesidad.

Por referencia a Rawls y su lista de derechos humanos cuyo respeto es condición de membrecía del Derecho de Gentes, resulta claro que en el caso de los refugiados estos derechos son vulnerados. Tal es el caso de derecho a la vida. Rawls considera que este derecho incluye lo necesario para la propia subsistencia y seguridad. Tanto en el caso de los refugiados en el sentido de la Convención, como de muchos refugiados medioambientales, pero también de los inmigrantes económicos que están por bajo el umbral suficientarista, se puede afirmar que no tienen lo necesario para su subsistencia y seguridad. En el caso de los refugiados el derecho a la libertad (esto es, ser libre de esclavitud, de trabajo forzado, una medida suficiente de libertad de conciencia, y que garantice la libertad de religión y pensamiento) también está comprometido, como lo está el derecho a la igualdad formal y en ocasiones la protección de grupos étnicos de genocidio. ${ }^{25}$

Por referencia al enfoque en las capacidades, en la versión de Nussbaum, resulta aún más evidente que la situación de los refugiados, los refugiados climáticos, pero también los económicos, implica una amenaza a su dignidad. Esto se debe a que este enfoque presenta demandas substantivas más exigentes que las teorías antes mencionadas. Considere la salud corporal, que refiere, entre otros, a la habilidad de tener buena salud, incluyendo salud reproductiva. $O$ el respecto a la integridad corporal, que refiere, entre otros, a tener las fronteras de nuestro cuerpo tratadas como soberanas, tener la habilidad de estar seguro contra asaltos, incluyendo asaltos sexuales, abuso sexual de niños y violencia doméstica, así como tener las oportunidades para satisfacción sexual y para elección en materias de reproducción. O el

25 Sin embargo, Rawls no considera que la migración deba ser considerada un asunto de justicia. Para un análisis y crítica inmanente de la posición rawlsiana frente a la migración, compare Loewe (2007a). 
respecto a sentidos, imaginación y pensamiento, que Nussbaum relaciona, entre otros, con la habilidad para evitar dolor innecesario. $\mathrm{O}$ el respecto a las emociones que refiere, entre otros, a no tener nuestro desarrollo emocional destruido por miedo y ansiedad, o por eventos traumáticos de abuso o descuido (Nussbaum 2000: 78-79). En todos estos casos, así como por referencia a las otras capacidades de su lista, la situación de los refugiados en el sentido de la Convención, pero también los climáticos y los económicos, es tal, que su posibilidad de tener una vida con dignidad humana está siendo amenazada. La integridad corporal, en todas sus descripciones, la habilidad para evitar dolor innecesario, pero también el respeto a las emociones y por consiguiente la amenaza de su destrucción por miedo, ansiedad y eventos traumáticos, están permanentemente amenazadas en el caso de todos ellos. Y no lo están sólo en el país de origen. Sino que lo están en las rutas de desplazamiento, bajo el dominio de mafias, con infraestructura deficitaria de transporte, y circunstancias climáticas y naturales. Pero también lo están en los países de destino, bajo el dominio de leyes en muchos casos draconianas, y dependiendo del arbitrio de los funcionarios.

\section{Tomando en serio la dignidad}

Si la dignidad implica el respeto debido a los seres humanos, la dignidad de los refugiados está siendo violada, sin que aquellos llamados a respetar la Convención estén a la altura de las exigencias y obligaciones que la dignidad impone. Pero también está siendo amenazada la dignidad de los refugiados climáticos y económicos, cuyas demandas no están cubiertas por la Convención. Si las condiciones de vida en el país de origen, la amenaza a la salud e incluso la amenaza vital de las rutas, así como, en ocasiones, las condiciones de acogida, vulneran la dignidad, la pregunta es quién o quiénes deben hacerse cargo.

Es común proponer que aquellos llamados a hacerse cargo son los países de origen. Desde esta perspectiva, la justicia se entiende como situada y local tanto en su justificación como alcance. Posicionamientos de este 
tipo son corrientes en el mundo comunitario. En el caso de la migración, Walzer ha defendido vehementemente la así llamada tesis de la asimetría (Walzer 1983). Esto es, la tesis aceptada en el derecho internacional mediante el reconocimiento de un derecho humano a emigrar, pero no de un derecho humano a inmigrar. Según el modelo argumentativo comunitario, el contexto histórico cultural, corrientemente entendido como delimitado por las fronteras políticas que definen al Estado-nación, da contenido a la justicia y determina su contexto de validez (Walzer 1983; Gray 2000; Rorty 1998). Por una parte, proteger la cultura política de un pueblo es proteger las bases constitutivas de lo justo. Ya que los inmigrantes con sus importaciones culturales pueden hacer peligrar la compresión compartida en que se basa la comunidad política cultural y así las bases constitutivas de lo justo, su recepción no se establece en términos normativos, sino que debe quedar al arbitrio de cada nación (Walzer 1983: 39). Por otra parte, las obligaciones de justicia apenas se extienden más allá de las fronteras políticas que circunscriben los contextos de entendimientos compartidos. En el caso de los inmigrantes, como la tesis de la asimetría claramente lo expresa, no hay una obligación de justicia para aceptar su ingreso, sino que su aceptación o rechazo descansa en los intereses de la sociedad, o expresa preceptos caritativos compartidos. La única excepción, según Walzer, serían los refugiados hacia los cuales hay obligaciones especiales o vínculos ideológicos o étnicos. ${ }^{26}$

Este tipo de posiciones están sujetas a críticas fuertes. Sólo mencionaré una de ellas: sociedades democráticas liberales reconocen valores y derechos fundamentales que incluso se expresan como derechos

\footnotetext{
${ }^{26}$ Por cierto, Walzer sí reconoce una moralidad de validez universal. Pero aquella es descrita de un modo extremadamente minimalista. Según esta concepción, habría una moralidad delgada (thin). Se trataría de una moralidad efectivamente compartida (no de una que se base en argumentos independientes), en el sentido de expresar el traslape de ideas que se derivan de moralidades densas (thick) específicas y contextualmente situadas particulares: "the moral minimum is not a free-standing morality. It simply designated some reiterated features of particular thick or maximal moralities" (Walzer 1994: 10). Pero en su interpretación, este mínimo moral no cubriría el caso de la migración, sino que implicaría obligaciones de justicia sólo en el caso de la esclavitud y el genocidio.
} 
humanos. Y una característica de estos derechos es que son universales. Por lo demás, el reconocimiento de estos derechos, al menos si se los entiende de un modo acotado, no es sólo privativo de las sociedades liberales. Como vimos, si seguimos la argumentación rawlsiana en su Derecho de Gentes, sociedades no liberales pero decentes se caracterizan por respetar ciertos derechos humanos fundamentales. Si estos derechos son parte del entendimiento político de un pueblo, entonces lo que se seguiría desde una perspectiva comunitaria, es que las demandas correspondientes debiesen tener un alcance universal. Por cierto, en las sociedades liberales los entendimientos compartidos no incluyen un derecho a inmigrar. Pero dado que estas sociedades valoran las libertades civiles y los derechos políticos, así como, en algunos casos, el derecho a subsistencia, considerándolos incluso como derechos humanos, ellas no pueden restringir la inmigración sin más cuando estos derechos y libertades están siendo amenazados. El mismo argumento lo podemos construir por referencia a la dignidad humana. La dignidad humana (entendida en base a un umbral) sería parte de los entendimientos compartidos de estas sociedades. Y como vimos, una característica de esta dignidad es su universalidad: todos los seres humanos tienen una demanda legítima a igual dignidad (es decir, a igual respeto). Si esto es así, entonces estos pueblos sí tendrían algún tipo de obligación normativa con respecto a la dignidad humana que sobrepasa sus fronteras políticas. Ya que en los casos que cubre la Convención son justamente los Estados de origen los que amenazan la dignidad, o son incapaces o no tienen la voluntad de evitar que esto suceda, resulta estéril anunciar que son ellos los que no cumplen con su responsabilidad. Evidentemente no la cumplen. Pero esto no quita ni urgencia ni fuerza a la demanda normativa de todos aquellos cuya dignidad está siendo amenazada por sus acciones u omisiones. En el caso de los refugiados medioambientales hay buenos argumentos para hacer descansar la responsabilidad, al menos parcialmente, en todos aquellos que son responsables y/o que se han beneficiado a costas del cambio climático (Loewe 2014). Sin embargo, si los responsables no se 
hacen cargo de los costos generados, mucho parece hablar a favor de que todos aquellos que tengan la capacidad lo hagan, reduciendo las situaciones de vulnerabilidad que amenazan la dignidad humana. Como vimos, incluso en un marco de argumentación comunitario, en base a los entendimientos compartidos, permitir la inmigración de aquellos cuya dignidad está siendo violada, sería una obligación de justicia de alcance global, al menos para las sociedades liberales, y quizás para las decentes. Este tipo de teorías debe aceptar que la protección de la dignidad humana de refugiados e inmigrantes potenciales constituye una razón válida para no rechazarlos sin más en las fronteras de estas sociedades, así como para facilitar su acceso, asegurar rutas, y acogerlos en condiciones que no amenacen su dignidad.

Hay otras estrategias argumentativas para justificar la exclusión de los inmigrantes. Algunas de ellas han sido articuladas por autores que defienden variantes del nacionalismo liberal (entre otros Miller 1995; Tamir 1993; Kymlicka 1989; 1995). ${ }^{27}$ Desde estas perspectivas, si bien habría razones para estipular la universalidad de algunas exigencias de justicia, tendríamos obligaciones fundamentales -en su materialidad y exigibilidad- hacia nuestros connacionales, que se antepondrían y que cualificarían de un modo relevante las obligaciones globales. Esto también se extiende al caso de la recepción de inmigrantes. Kymlicka y Miller, por ejemplo, aceptan la existencia de ciertas obligaciones globales de justicia, pero defienden la restricción al acceso de inmigrantes, tornando obligatoria su recepción sólo cuando no se cumplen las obligaciones globales que ayudan a los inmigrantes potenciales en su lugar de origen. Desde esta perspectiva, la respuesta correcta a la presión migratoria no implica su recepción, sino que el mejoramiento de sus condiciones de vida en los países de origen (Kymlicka 1995; Miller 1999; 2000; 2007). Kymlicka ha presentado un argumento para defender la prioridad de las obligaciones

${ }^{27}$ En otro lugar he discutido la compleja posición del nacionalismo liberal de Kymlicka con respecto a las teorías comunitarias señaladas en el punto anterior (Loewe 2006). 
hacia los connacionales. Según este argumento, un contexto cultural "rico" y "seguro" es necesario para posibilitar la autonomía de los individuos (Kymlicka 1989; 1995). Por tanto, la pertenencia cultural debiese ser entendida como un bien primario rawlsiano (Kymlicka 1989: 167), ya que nos ofrece opciones y criterios de valoración, ambos necesarios para poder formar, revisar y perseguir la propia concepción del bien. El contexto de elección sería una "cultura societal", caracterizada, entre otros, por una lengua y una historia compartida. Ya que en la modernidad las culturas societales tomarían la forma de la nación, la protección de la autonomía implicaría proteger la cultura nacional. ${ }^{28} \mathrm{Al}$ provenir de otras culturas, los inmigrantes pueden hacer peligrar la riqueza y seguridad del contexto de elección y así la autonomía personal de los connacionales. ${ }^{29}$

Pero esta tesis no se sostiene. En primer lugar, si bien es correcto que se requieren opciones y criterios de valoración para tomar decisiones inteligentes, es incorrecto afirmar que estos responden a una gramática nacional (Waldron 1995). Así, aceptar la migración por razones de justicia no implica quitarle sustento a la capacidad autonómica. Segundo: aunque fuese el caso que el desarrollo de la autonomía requiriese de un contexto cultural rico y seguro, lo que se seguiría es que no todos la harían peligrar, sino sólo aquellos inmigrantes que provienen de culturas muy distintas, y además que no están dispuestos a aceptar y respetar la cultura de la nación receptora. Además, si se trata de culturas societales caracterizadas por la diversidad cultural, no se sigue que la protección de la autonomía esté mediada por la restricción de la inmigración.

Si contrastamos estos resultados con la aceptación por parte de estas teorías de algún tipo de obligación de justicia global, resulta evidente que aceptar inmigrantes sí puede ser un mecanismo para hacerse cargo de

\footnotetext{
${ }_{28}$ Una crítica al argumento de Kymlicka en Loewe (2007b).

29 Otros argumentos contra fronteras porosas en el caso de los inmigrantes, refieren al estado social que la identidad nacional posibilitaría (Miller 1995), y a la lealtad nacional que involucra el gran proyecto nacional intergeneracional (Tamir 1993). En este texto no me referiré a estos argumentos. Compare Loewe (2018).
} 
estas obligaciones, ${ }^{30}$ al menos toda vez que no es posible cumplir estas obligaciones de otro modo. $Y$ este es exactamente el caso en todas las situaciones que he examinado en este artículo: los casos de los perseguidos, de los refugiados climáticos, así como de muchos inmigrantes económicos, corresponden a situaciones en que la dignidad humana está siendo amenazada de tal modo, que no es razonable esperar que los mecanismos de la justicia distributiva global sean efectivos, al menos no para todos aquellos que actualmente se encuentran en tal situación de vulnerabilidad. Probablemente cuando los mecanismos de la justicia global sean efectivos, en caso que lleguen a serlo, todos aquellos cuya dignidad está siendo violada, ya estarán muertos.

\section{6) Conclusiones}

De acuerdo al argumento elaborado, la dignidad no se basa en propiedades naturalistas o supraempíricas, sino que es el resultado del respeto y reconocimiento mutuo. $Y$ este respeto y reconocimiento mutuo implica reconocer la condición de vulnerabilidad en que se encuentran los seres humanos. Así, la dignidad se puede perder - cuando las condiciones son indignas para la vida humana-, pero la demanda legítima de cada cual a que se respete su dignidad permanece. Esta concepción admite interpretaciones diversas con respecto al tipo de exigencias que caracterizan a la dignidad humana. En este texto la he interpretado por referencia a una concepción suficientarista. A modo de ejemplo, recurrí a la defendida por Margalit al identificar tres modos de humillación, a la defendida por Rawls con su concepción minimalista de derechos humanos, y a la interpretación de Nussbaum del enfoque en las capacidades. Por referencia a todas estas teorías, la dignidad de los refugiados, de los refugiados climáticos o medioambientales, pero también de muchos inmigrantes económicos,

30 Una discusión acerca de si en el caso de la migración debiese primar una política de fronteras abiertas o políticas redistributivas de justicia global, en Loewe (2012). 
está siendo amenazada o llanamente violada. Y dado que la demanda de la dignidad se establece en términos universales e igualitarios, la obligación de no sólo respetarla, sino también la obligación positiva de realizarla, se extiende más allá de las fronteras nacionales. Esto implica una obligación no sólo de aceptar el ingreso de aquellos refugiados e inmigrantes cuya dignidad esté siendo violada, sino también de asegurar las rutas de un modo acorde a esta dignidad, y de diseñar los mecanismos administrativos de recepción y otorgación de estatus de un modo tal que no la ponga en entredicho. Como examinamos, los cuestionamientos de las teorías comunitarias, pero también de las nacionalistas liberales, a esta afirmación son débiles y a su vez cuestionables. Las referencias ubicuas e inflacionarias a la dignidad humana, como categoría declarativa de buenas intenciones, debiesen ceder lugar a políticas que efectivamente la tomen en serio. ${ }^{31}$

\section{Bibliografía}

Barry, Brian; Gooding, R. (eds.) (1992): Free movement: ethical issues in the transnational migration of people and money. Pennsylvania: Penn State University Press.

Brown, Lester (2011): World on the edge: how to prevent environmental and economic collapse. Earth Policy Institute, New York, London: W.W. Norton \& Company (http://www.earth-policy.org/images/uploads/book_files/ wotebook.pdf)

Carens (1992): “Migration and morality: a liberal egalitarian perspective”, en Barry/ Gooding (eds.): 25-47

Carens, J. (1987): "On Aliens and citizens. The case of open borders". Review of Politics, IL/ 2.

Debus, Tesa; Kreide, Regina; Krennerich, Michael; Malowitz, Karsten; Pollmann, Arnd; Zwingel, Susanne (eds.) (2010): número “Philosophie der Menschenwürde" en Zeitschrifft für Menschenrechte, 1, Wochenschau Verlag.

31 Una política en esta dirección, sería la movilidad libre. Para defensas de esta posición compare Carens (1987; 1992); Velasco (2016); Loewe (2007a; 2012); una defensa condicionada, en Shachar (2009), una crítica de esta defensa en Loewe (2018a). 
Dummett, Michael (2001): On immigration and refugees, London, New York: Routledge.

El-Hinnawi (1985): Environmental Refugees. Nairobi: United Nations Environmental Programme.

Gosepath, Stefan/ Lohman, Georg (eds.): Philosophie der Menschenrechte, Suhrkamp, Frankfurt am Main.

Gray, Clark (2010): Environmental refugees or economic migrants? Population Reference Bureau (http://www.prb.org/Publications/Articles/2010/environmentalmigrants.aspx)

Gray, John (2000): Two faces of liberalism. Cambridge: Polity Press.

International Migration Report 2015. (http://www.un.org/en/development/desa/population/migration/publications/migrationreport/docs/ MigrationReport2015.pdf)

Kymlicka, W. (1995): Multicultural citizenship. Oxford: Oxford University Press.

Kymlicka, Will (1989): Liberalism, community and culture. Oxford: Clarendon Press.

Kymlicka, Will (ed.) (1995): The rights of minority cultures. Oxford: Oxford University press.

Loewe, Daniel (2006): “Un nouveau communautarisme culturel” en: Merle, J-Ch.; Niquille, J.; Schumacher, B. Figures du communautarisme. Herzogenrath: Shaker Verlag.

Loewe, Daniel (2007a): “Inmigración y el derecho de gentes de John Rawls. Argumentos a favor de un derecho a movimiento sin fronteras". Revista de Ciencia Política, Santiago, v. 27/2: 23-48.

Loewe, Daniel (2007b): "Teorías de justicia igualitaria y derechos culturales diferenciados". Isegoría. 36: 275-302.

Loewe, Daniel (2008): "Liberalismo igualitario e igualitarismo orientado al bienestar: las demandas de las minorías culturales", Signos Filosóficos, vol. X, núm. 20, julio-diciembre, 2008, pp. 25-60.

Loewe, Daniel (2009): "El enfoque en las capacidades y las demandas por derechos culturales", Signos Filosóficos, México, Vol.11/2, pp. 103-146.

Loewe, Daniel (2010a): "Los náufragos de nuestro tiempo. El caso de los refugiados". Arbor, 186:744: 555-570.

Loewe, Daniel (2010b): “Liberalismo, inmigración y justicia global: Obligaciones especiales hacia nuestros conciudadanos". Isegoría, no. 43: 435-458. 
Loewe, Daniel (2010c): “Obligaciones hacia las generaciones futuras. El caso contractual”, Veritas 55/1: 21-66.

Loewe, Daniel (2011): “Der Umfang der moralischen Gemeinschaft: Einbezug nichtmenschlicher Tiere in einen vertragstheoretischen Argumentationsrahmen". En: Kovács, L./ Brand, C. (eds.): Forschungspraxis Bioethik. Freiburg, München. Alber Verlag.

Loewe, Daniel (2012): “Obligaciones de justicia: ¿open borders o justicia Distributiva?" Arbor, Vol 188, No 755

Loewe, Daniel (2014): "Refugiados climáticos: ¿quién debe cargar los costos?" REMHU - Rev. Interdiscip. Mobil. Hum., Brasília, Ano XXII, n. 43, pp: 169-187.

Loewe, Daniel (2015a): “Cuán liberal es la teoría de las relaciones internacionales de Rawls?". Veritas 60 (1): e1-e35.

Loewe, Daniel (2016): “Contractualismo y animales: ventajas mutuas e imparcialidad", en: Figueroa/ Widow (eds.): Ética Filosófica. Aproximaciones contemporáneas. Ril editores.

Loewe, Daniel (2018a): “Justicia global, impuesto a la ciudadanía y fronteras abiertas" Hybris 9.

Loewe, Daniel (2018b): "Justicia distributiva, comunidades nacionales, inmigrantes y fronteras abiertas” Anales, Universidad de Chile.

Lohmann, Georg (1999): "Menschenrechte zwischen Moral und Recht", en Gosepath, Stefan/ Lohman, Georg (eds.)

Luhmann, Niklas (1965): Grundrechte als Institution, Berlin.

Margalit, Avishai (1997): The decent Society, Harvard University Press.

Martel, Yann (2001): Life of Pi, Random House of Canada.

Milanovic, Branko (2016): Global inequality. A new approach for the age of globalization. Belknap Press.

Miller, David (1995): On Nationality. Oxford University Press.

Miller, David (1999): “Justice and inequality”. En Hurrell/ Wood (eds.): Inequality, globalization, and world politics. Oxford, Oxford University Press.

Miller, David (2000): Citizenship and National Identity. Cambridge, Polity Press.

Miller, David (2007): National responsibility and global justice. Oxford, Oxford University Press. 
Minura, Nobuo et al (2007): Small Island, en: Parry, Martin; Canziani, Osvaldo; Palutikof, Jean; Van der Linder, Paul; Hanson, Clair (eds.): Impacts, adaptation and vulnerability. Contribution of the Working Group III to the Fourth Assessment Report of the Intergovernmental Panel on Climate Change. Cambridge: Cambridge University Press.

Myers, Norman (2005): Environmental Refugees: An emergent security issue. 13th Economic Forum, 2005. (http://www.osce.org/eea/14851?download=true).

Nussbaum, Martha (2000): Women and human development, Cambridge, UK, Cambridge University Press.

Nussbaum, Martha (2006): Frontiers of justice, Cambridge MA: Harvard University Press.

ONU. Declaración Universal de Derechos Humanos, 1948.

Rawls, J. (1971): A theory of justice. Harvard: Harvard University Press.

Rawls, J. (1993a): Political liberalism. New York: Columbia University Press.

Rawls, J. (1999): The law of peoples with "The idea of public reason revisited. Harvard: Harvard University Press.

Rorty, R. (1989): Achieving our country. Harvard: Harvard University Press.

Schiller, Friedrich (1962): Sämtliche Werke, Band 1, München.

Sen, Amartya (1999): Development as Freedom, Nueva York: Anchor Books.

Shachar, Ayelet (2009): The Birthright Lottery. Citizenship and Global Inequality. Mass.: Harvard University Press.

Singer, Peter (1993): Practical Ethics, Cambridge: Cambridge University Press.

Stern, Nicholas (ed.) (2006): The Economics of Climate Change: The Stern Review. Cambridge: Cambridge University Press.

Talbert, Matthew (2006): "Contractualism and Our Duties to Nonhuman Animals", Environmental Ethics 28: 201-215.

Tamir, Yael (1993): Liberal nationalism. Princeton: Princeton University Press. Tugendhat, Ernst (1995): Vorlesungen über Ethik, Frankfurt/M.

Velasco, Juan Carlos (2016): El azar de las fronteras, Fondo de cultura económica. Vitale, Ermanno (2006): Ius Migrandi, Melusina. 
Waldron, Jeremy (1995): "Minority cultures and the cosmopolitan alternative". En: Kymlicka (ed.): Multicultural citizenship. Oxford: Oxford University Press. Waldron, Jeremy (2014): The harm in hate speech. Harvard University Press. Waldron, Jeremy (2015): Dignity, Rank, and Rights. Oxford University Press. Walzer, M. (1983): Spheres of justice: a defense of pluralism and justice. New York: Basic Books.

Walzer, M. (1994): Thick and thin. Notre Dame: University of Notre Dame Press. Wetz, Franz Josef (1998): Die Würde der Menschen ist antasbar. Klett-Cotta, Stuttgart. 